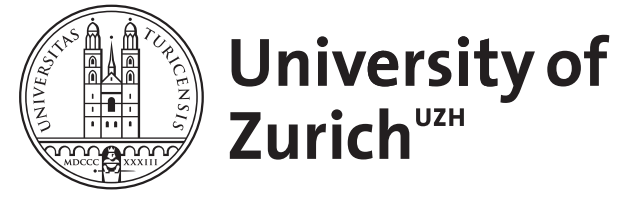

\title{
Analysis of movement data
}

Dodge, Somayeh ; Weibel, Robert ; Ahearn, Sean C ; Buchin, Maike ; Miller, Jennifer A

DOI: https://doi.org/10.1080/13658816.2015.1132424

Posted at the Zurich Open Repository and Archive, University of Zurich ZORA URL: https://doi.org/10.5167/uzh-130639

Journal Article

Accepted Version

Originally published at:

Dodge, Somayeh; Weibel, Robert; Ahearn, Sean C; Buchin, Maike; Miller, Jennifer A (2016). Analysis of movement data. International Journal of Geographical Information Science, 30(5):825-834.

DOI: https://doi.org/10.1080/13658816.2015.1132424 


\section{Analysis of Movement Data}

Somayeh Dodge ${ }^{1^{*}}$, Robert Weibel ${ }^{2}$, Sean C Ahearn ${ }^{3}$, Maike Buchin $^{4}$, Jennifer A. Miller ${ }^{5}$

${ }^{I}$ Department of Geography and Environmental Studies, University of Colorado Colorado Springs, USA

${ }^{2}$ Department of Geography, University of Zurich, Switzerland

${ }^{3}$ Department of Geography, City University of New York - Hunter College, New York, USA

${ }^{4}$ Faculty of Mathematics, Ruhr University Bochum, Germany

${ }^{5}$ Department of Geography and the Environment, The University of Texas at Austin, USA

"Corresponding author's e-mail: somayeh.dodge@gmail.com

\section{Introduction}

The study of movement is progressing rapidly as a sub-discipline in Geographic Information Science (GIScience). At the fulcrum of this new research area in GIScience are movement observations. Movement observations may be understood as spatiotemporal signals, which carry information on the movement of dynamic entities and the underlying mechanisms that drive their movement. These observations are key to the study and understanding of movement. Technological advancements in global positioning systems (GPS) and related satellite tracking technologies have resulted in significant increases in the availability of highly accurate data on moving phenomena, 
dramatically outpacing the development of appropriate methods with which to analyze them. In addition to increased spatial accuracy and temporal resolution of the locational information, improvements are being made to accelerometers and 'biologgers' that enable the collection of ancillary behavioral and physiological information.

This special issue emerged from a pre-conference event associated with the GIScience 2014 conference held in Vienna: a workshop organized by the authors on "Analysis of Movement Data" (AMD 2014). The workshop and this special issue explore recent trends in the study of movement and novel methods for analyzing and contextualizing movement data. A broad range of topics is covered concerning movement analysis, representation and modeling. The studies presented use movement data from different domains, such as transportation (vehicles, marine traffic), cyclists and athlete tracking data, storm events, and movement ecology (birds, mammals, etc.).

This editorial intends to frame and position the papers included in this special issue and to provide recommendations for future directions in the analysis of movement data. In order to frame the work presented here, we use the overarching research framework for the study of movement proposed by Dodge (2015). This framework, shown in an adapted version in Figure 1, posits that the study of movement consists of a continuum of research ranging from understanding movement to construct knowledge of the behavior of dynamic objects, to using this knowledge for modeling and prediction of movement. Visualization facilitates this process through data exploration, hypothesis generation, and communication of the outcomes (Wood et al. 2011, Andrienko et al. 2013, Zhang et al. 2013, Xavier and Dodge 2014). The framework relies on an iterative validation process, where analytics and models are parameterized, calibrated, and improved using real movement observations. 


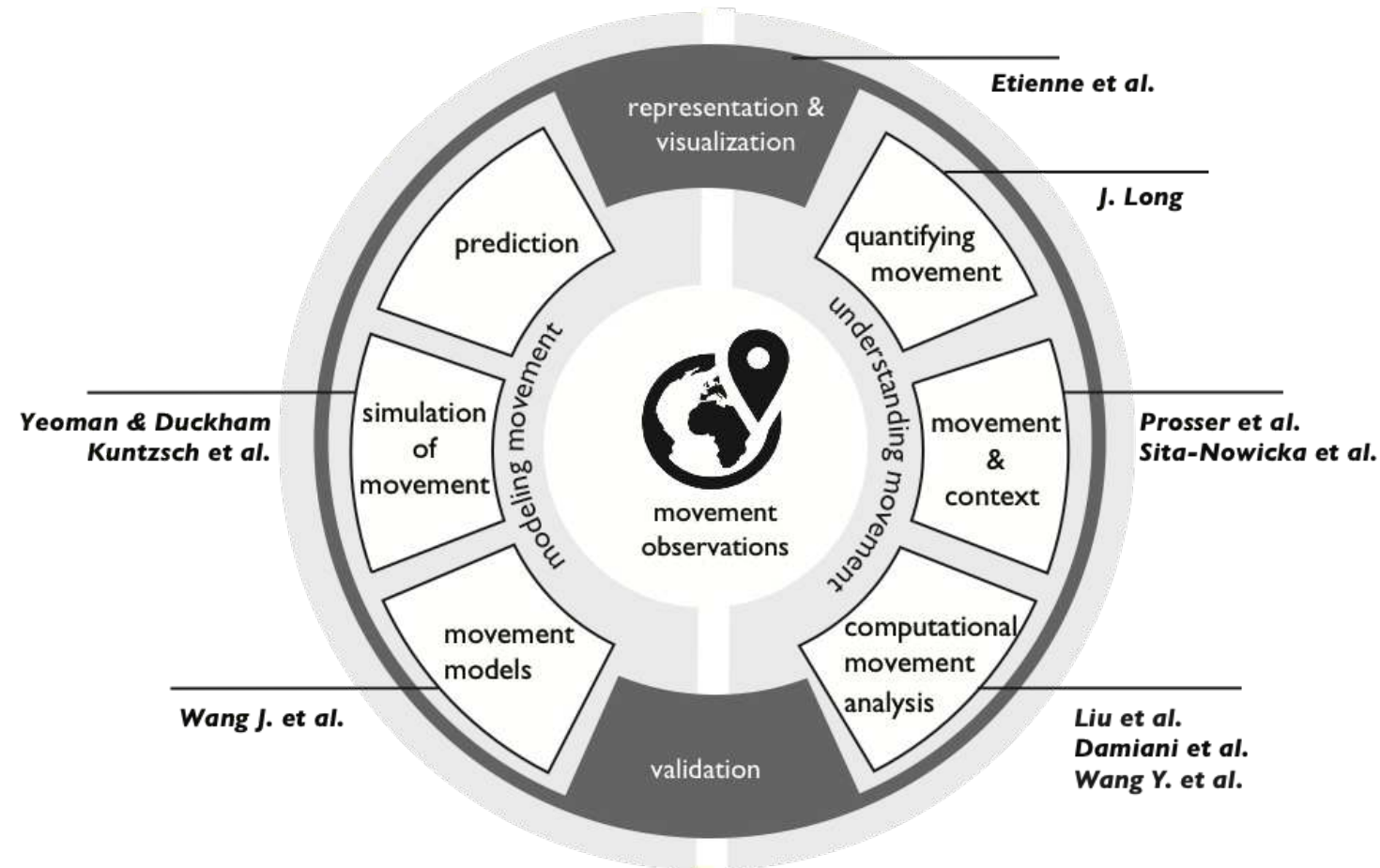

Figure 1. Articles presented in this special issue cover a range of research for understanding and modeling of movement (modified version of the continuum from Dodge 2015)

Understanding movement, shown on the right side of Figure 1, entails development of methods for quantification of movement and its parameters (Dodge et al. 2008, Long and Nelson 2012, Laube 2014, Demšar et al. 2015); analysis of its context and external influences on movement (Schick et al. 2008, Andrienko et al. 2011, Dodge et al. 2013, Buchin et al. 2014, Miller 2015); and detection of movement patterns through computational movement analysis (Long and Nelson 2012, Laube 2014, Purves et al. 2014, Soleymani et al. 2014). Modeling movement, shown on the left side of Figure 1, consists of creating models, developing simulations and making predictions (Ahearn et al. 2001, Hornsby and Egenhofer 2002, Schick et al. 2008, Torrens et al. 2012, Song and Miller 2014, Technitis et al. 2015). Thus, the culmination of this continuum (Figure 1) is the building of movement models given a set of 
conditions and the creation of simulation systems to predict movement and state changes in dynamic systems. This final step is a departure from a mere analysis of movement patterns, to the understanding of the processes underlying movement. Through this understanding we are able to build models that adequately represent the real world systems. Equally - and this is reflected by the circular arrangement of Figure 1 - this 'final' step also marks the beginning of an iteration, informed by a consolidated level of understanding that improves our knowledge about the processes that we are studying.

\section{The papers in this special issue}

Figure 1 is used as a framework for positioning the papers of this special issue, marked in bold italics. The articles of this issue range from developing analysis and computational approaches in order to understand movement processes (i.e. the right side of the continuum in Figure 1) to visualization or representation of movement data (i.e. the ether of the continuum), to modeling of dynamic objects (i.e. the left side of the continuum).

\subsection{Understanding Movement}

Movement data can inform us about the behavior of dynamic phenomena and the underlying mechanisms driving movement. Due to its complex nature, understanding movement and constructing knowledge from movement data requires sophisticated tools and methods to represent, quantify, contextualize, and analyze movement trajectories. As documented in this section, the main focus of most papers in this special issue lies in understanding of movement. 


\subsubsection{Representation and visualization of movement}

In their article, Etienne et al. propose a novel representation for a spatio-temporal pattern called the trajectory box plot (TBP), which adapts the concept of the classical box plot for trajectory data. In this article, the TBP method is applied to a cluster of trajectories with the same itineraries to represent the reference median trajectory of the cluster and trajectory variations in the cluster. TBPs are useful to summarize and analyze trajectory streams, understand their spatiotemporal density and detect outliers. The article illustrates the benefits of this method through visual analysis of a large set of ship trajectories (over 500 tracks) from a real-world scenario.

\subsubsection{Quantifying movement}

One of the foremost tasks in analyzing movement is to quantify movement by constructing movement trajectories and computing associated movement parameters. Often movement data include missing tracking points, gaps, and irregular sampling intervals. The challenge here is to interpolate movement data to construct smooth and continuous trajectories for further analysis. Long introduces a new method for movement trajectory interpolation called kinematic interpolation. Kinematic interpolation incorporates object kinematics (i.e., velocity and acceleration) into the interpolation process. In a comparative study, kinematic interpolation is evaluated against other interpolation algorithms such as linear interpolation, constrained random walk, Bézier curves, and Catmull-Rom curves using six empirical datasets (trajectories of two types of correlated random walks, caribou, cyclist, hurricane, and athlete tracking data). The results show kinematic interpolation is a promising interpolation method for fast moving objects (e.g., the cyclist, hurricane, and athlete tracking data). 


\subsubsection{Movement and context}

Movement does not take place by itself. Information on the context in which it occurs, such as the nature of interactions of moving phenomena with their environment and with other individuals, can facilitate a better understanding of movement. The article by Prosser et al. is a representative of one of the major application domains of the analysis of movement data: movement ecology. More specifically, that article reports on the first movement study in which free-grazing domestic ducks were tracked to estimate the potential for domestic ducks from farms located near natural wetlands to come in contact with migratory wild waterfowl, thus increasing the risk of disease transmission, including highly pathogenic avian influenza H5N1. GPS dataloggers were fitted to freegrazing domestic ducks and the dynamic Brownian bridge movement model (dBBMM) was used to estimate the utilization distribution of ducks, that is, their habitat use. This was used to identify the relative use of areas that are shared by both domestic and wild birds which may have the highest risk of disease transmission between the two populations.

In a different application, Sita-Nowicka et al. present an integrated approach to analyze human mobility using a combination of tracking data obtained from volunteered GPS trajectories and contextual spatial information obtained from surveys. The contextual data used in this study provide a description of the spaces that people move through. Through data mining and spatio-temporal analysis, their approach is to apply trajectory segmentation to identify behaviors associated with travel segments (i.e. moves) and significant places (i.e. stops). The article investigates how travel modes depend on the residential location, age or gender of the tracked individuals. The article proposes a novel methodology for capturing "third places" (i.e. places of interest that are beyond main home/work locations where individuals spend time to socialize) through the identification of typologically relevant significant places ordered by visit 
duration. Their methodology is evaluated using a collection of trajectories from 205 volunteers linked to contextual spatial information on the types of places visited and the transport routes they use.

\subsubsection{Computational movement analysis}

Computational movement analysis, a term coined by Laube (2014), relates to computational methods to detect patterns structures in movement data. These methods provide the foundation to developing insight into the behavior of moving phenomena from raw movement data.

The paper by Damiani et al. deals with the semi-automated detection of partial migration movement patterns in animal ecology. Partial migration refers to more individual-based movement that does not result in a clear spatial and temporal separation of ranges and it is therefore more difficult to measure than migration. Here the authors test a relatively new temporally explicit density based clustering algorithm (SeqScan) that extends traditional clustering algorithms. They suggest that its ability to measure the fine-scale variability between resident and migratory movement makes it particularly useful for analyzing partially migratory individuals. SeqScan allows a 'noise' component to be separated from the data that absorbs a partially migratory individual's excursions outside of the core region as well as a stationarity index to measure the level of mobility associated with a cluster in order to determine whether the movement trajectory corresponds to resident or migratory movement. Using movement data on two large herbivores (roe deer and red deer) in Bavaria, computational movement analysis is employed to classify movement trajectories into clusters and excursions. These trajectory classifications allow for finer-scale distinctions among movement patterns and can subsequently be used to infer similarly fine-scale behaviors associated with them. 
Through a similar application, Wang Y. et al. propose the notion of continuous behavior patterns as a concise representation of animal migration routes. They develop a framework to derive these patterns from raw trajectories and evaluate their methodology on tracking data sets of two species of wild birds: bar-headed geese and ruddy shelducks. The patterns found in the experiments allow identifying different behavioral stages and associated functional areas, such as stopovers, in an ecologically insightful manner. Furthermore, the collective analysis across marked individuals may highlight the movement differences among individuals to the population-level. Overall the experiments demonstrate the potential of the method for discovering spatiotemporal semantics of collective animal migration behaviors.

Liu et al. propose a directed spatiotemporal graph model to represent the dynamic characteristics of storm events. In the model, storm objects are represented as nodes of the graph, and interactions of storm objects as edges. Graph algorithms are explored to generalize and analyze movement events using time series of spatial snapshot data. The proposed method is applied to storm events inferred from weather radar reflectivity images of a severe storm outbreak in the US in April 2011. The storm events identified in the experiments are consistent with previous studies, and the method shows potential for capturing a more detailed climatology of precipitation characteristics. Hence the work suggests that the event approach provides an effective means to analyze dynamic behaviors existing in time series of spatial snapshot data.

\subsection{Modeling movement}

\subsubsection{Movement models}

Studies in movement analysis today use either the Lagrangian or the Eulerian approach to movement data collection. In the Lagrangian approach the movement information 
that is collected is referenced to the moving object (e.g. by fitting a GPS device to the object, or by video-tracking the object as it moves). The Eulerian approach focuses on movement information that is referenced to fixed locations, such as Bluetooth beacons, WLAN hot spots, or mobile phone cell towers. Both approaches obviously do co-exist, but little work has been done so far that links both of these fundamental and dichotomous paradigms. In their paper in this special issue, Wang J. et al. are contributing to filling this void. First, they develop the formal foundations for the two conceptual movement models, linking these to the object-field dichotomy, as well as the SNAP/SPAN ontologies of dynamic features. Based on this formal framework, they show conceptually and experimentally how data that have been captured with respect to one paradigm (either Lagrangian or Eulerian) can be transformed into the other paradigm. One of the key findings of this work is to highlight the critical role of the spatial and temporal granularity of movement data in controlling the accuracy of these transformations.

\subsubsection{Simulation of movement}

Emerging sensor technologies, such as mobile geosensor networks (mGSNs), offer potentially new ways to monitor and analyze dynamic systems and spatiotemporal phenomena. These sensor networks require new decentralized spatial computing (DeSC) algorithms to detect movement patterns, such as convoys. Convoys are densitybased movement patterns that are defined as a set several moving objects remaining in close proximity of each other for a duration of time. In their article, Yeoman and

Duckham describe their research into designing, simulating and evaluating DeSC algorithms. This article demonstrates decentralized detection and monitoring approaches for convoy patterns with computation occurring in the network, without central control and without quantitative positioning. An empirical evaluation in agent- 
based simulations confirms effectiveness and efficiency characteristics of the algorithms, and illustrates the utility of such approaches in studying and characterizing decentralized algorithm behaviors in complex dynamic systems.

Kuntzsch et al. aim at geometric and topologic reconstruction of traffic networks from GPS trajectories through a multi-step simulation process. Their approach combines heuristic techniques (to obtain road segments between junctions by kernel density-based aggregation) with generative modeling to reconstruct optimal junction geometries. The results are validated using benchmark data sets of several cities of different size (e.g. Chicago, Berlin, Athens). This work contributes to 'automatic map construction methods' based on ubiquitous mobile tracking data.

\section{Applications of Movement Research}

As evidenced in Section 2, research on movement applies to many areas of science and technology such as transportation, human mobility, behavioral studies, environmental studies, and movement ecology, to name but a few. The articles in this special issue are representative of different application domains, where analytic methods and models from GIScience are developed to study and understand movement in various settings.

Detection of movement patterns is essential in many applications including transportation and understanding of human dynamics (Batty et al. 2003, Torrens et al. 2012). In this special issue, the papers by Sita-Nowicka et al., Yeoman and Duckham, and Kuntzsch et al. look into different aspects of human mobility and transportation systems through the study of movement. Etienne et al. examine transit trajectory data (in this case ship movements) with similar itineraries through a visual analytics approach.

Movement ecology has recently emerged as a general paradigm that interlinks research on how, why, when and where animals, plants and microorganisms move 
(Nathan et al. 2008; Nathan \& Giuggioli 2013). In this special issue, Damiani et al. and Wang Y. et al. apply movement analytic methods to investigate migration patterns and local movement of animals. Prosser et al. study the interactions of moving individuals (in this case domestic ducks) with their environment and the chances of contacts with wild birds to estimate the risk of disease transmission to the human environment. This area of movement research can have significant implications in public health and understanding of the spread of diseases and exposure to infections or pollution (Theophilides et al. 2006, Lu and Fang 2014).

In environmental studies and mitigation of natural hazards, the study of movement is key to modeling the development patterns of hurricanes, oil spills, and wildfires. The article by Liu et al. represents such an application of movement research in environmental studies through the analysis of storm events.

\section{Recommendations for future directions}

In GIScience, movement research extends previous work in time geography that focused primarily on representation and semantics. Clearly, however, the methodological and analytical framework associated with this research area is still evolving. To conclude this editorial, we would like to point out some of the issues that future research should address.

\subsection{Collaboration with domain sciences}

This special issue covers a range of studies on understanding and modeling movement in a variety of applications (i.e. human mobility, movement ecology, and environmental studies). As recent years have shown, some of the very best results have been generated from research that was carried out in close collaboration between GIScientists and research from application domains. This has for instance been vividly 
shown in the European research network MOVE (www.move-cost.info; Purves et al. 2014; Demšar et al. 2015). In fact, we are of the opinion that real progress in research can only be made if researchers from the methods-oriented sciences (GIScience, computer science) are willing to engage in the research questions of the domain specialists. The reward for this will be that the methods-oriented researchers get access not only to precious data but also to precious domain knowledge. Beyond the 'classical' application domains (animal ecology, transportation, human mobility), one area where we see particular potential for future research is human health. Examples include, the study of the exposure of humans to infectious diseases and pollution sources, and the space-time dynamics of genetic illnesses.

\subsection{Taking into account context}

As also documented here, the main emphasis of movement research so far has been on the development of methods to understand behavior of moving phenomena through analysis of movement observations (the right side of the continuum in Figure 1). In contrast to much of the previous research, however, the papers of this issue also manifest a growing attention to the relationships between movement and its underlying context, the external factors that influence movement, and the interactions of moving individuals with their environment and with other individuals. Movement is a complex combination of the behavior of the individual and these contextual factors that drive or constrain movement. Thus, a deeper understanding of patterns of movement can only be achieved through analysis and modeling of movement given these factors.

\subsection{From analysis to simulation and prediction}

As also evidenced in this special issue, although methods for analysis of movement data are progressing rapidly, development of simulation and predictive 
models for movement, as well as effective visualization techniques to represent dynamic phenomena are lagging behind.

Existing modeling approaches such as time-geography models, random walks, and Brownian Bridge models are often employed as standard movement models. New research is required to re-visit the capacities and limitations of these models or to develop new simulation models for capturing complex movement behaviors across various spatial and temporal scales and within different contexts. These models need to be informed, calibrated and validated by real movement observations and be able to predict the response of the agent to a changing environment.

\subsection{Working through the scales}

Movement data today are captured at increasingly finer temporal sampling intervals. Movement processes, however, are typically characterized by multiple behavioral modes in the movement path of an individual that are expressed at different spatial and temporal scales. Simply confining the analysis scale to the original fine temporal granularity will overlook the fact that different movement patterns are evident at different spatiotemporal scales and that each movement pattern has a particular scale range at which it is manifested. Thus, extracting behaviors from movement data requires an analysis approach working at multiple scales — essentially a cross-scale approach — to establish the appropriate scale for each particular movement pattern, and to establish the transitions between behavioral modes.

\subsection{Multi-sensor measurement and analysis}

New technologies provide valuable multidimensional views of real word phenomena and the processes that drive them. Examples of these multiple sensor technologies and data sources include GPS, accelerometers, mobile phone positioning 
data, physiological parameters measures e.g. in sports tracking devices, remote sensing of the environment (e.g. for weather conditions, or landscape information). These heterogeneous datasets are obtained in different formats and structure, at different spatial and temporal granularities. The integration of these diverse data types requires methods to synchronize and integrate the measurements in order to capture a more complete picture of the behavior of the moving object and in the system in which it operates.

\subsection{Moving towards massive data}

Many of current movement studies employ only a small dataset of tracking observations. With recent advances in sensor and mobile technologies, movement data are growing in granularity and volume, and are collected continuously in real-time. Inevitably, future research should look into the scalability of correct methods, and develop new analytics for big movement datasets and tracking data streams.

\subsection{Visualizing movement}

We have moved from visualization of spatial phenomena to representation of spatiotemporal phenomena using techniques such as animation, space-time cubes, and multivariate representations. Visualization not only facilitates understanding of movement and discovery of unexpected patterns, it is an integral component of dynamic simulation systems. Therefore, more research is required on the development of new and effective methods to represent movement in relation to environment and context.

\section{References}

Ahearn, S.C., David, J.L., Joshi, A.R., and Ding, J., 2001. TIGMOD: an individualbased spatially explicit model for simulating tiger / human interaction in multiple use forests, Ecological Modelling, 140, 81-97.

Andrienko, G., Andrienko, N., Bak, P., Keim, D., and Wrobel, S., 2013. Visual 
Analytics of Movement. Berlin, Heidelberg: Springer Verlag.

Andrienko, G., Andrienko, N., and Heurich, M., 2013. An event-based conceptual model for context-aware movement analysis. International Journal of Geographical Information Science, 25, 1347-1370. doi:10.1080/13658816.2011.556120.

Batty, M., Desyllas, J., and Duxbury, E., 2003. The discrete dynamics of small-scale spatial events: agent-based models of mobility in carnivals and street parades. International Journal of Geographical Information Science, 17 (7), 673-697.

Buchin, M., Dodge, S., and Speckmann, B., 2014. Similarity of trajectories taking into account geographic context. Journal of Spatial Information Science, 9 (9), 101124.

Demšar, U., Buchin, K., Cagnacci, F., Safi, K., Speckmann, B., Van de Weghe, N., Weiskopf, D., and Weibel, R., 2015. Analysis and visualisation of movement: an interdisciplinary review. Movement Ecology, 3 (1), 1-24.

Dodge, S., 2015. From Observation to Prediction: The Trajectory of Movement Research in GIScience. In: H. Onsrud and W. Kuhn, eds. Advancing Geographic Information Science: The Past and Next Twenty Years.

Dodge, S., Bohrer, G., Weinzierl, R., Davidson, S.C., Kays, R., Douglas, D., Cruz, S., Han, J., Brandes, D., and Wikelski, M., 2013. The environmental-data automated track annotation (Env-DATA) system: linking animal tracks with environmental data. Movement Ecology, 1 (1), 3.

Dodge, S., Weibel, R., and Lautenschütz, A.-K., 2008. Towards a taxonomy of movement patterns. Information Visualization, 7 (3-4), 240-252.

Hornsby, K. and Egenhofer, M.J., 2002. Modeling Moving Objects over Multiple Granularities. Annals of Mathematics and Artificial Intelligence, 36, 177-194.

Laube, P., 2014. Computational Movement Analysis. Springer International Publishing.

Long, J.A. and Nelson, T.A., 2013. A review of quantitative methods for movement data. International Journal of Geographical Information Science, 27(2), 292-318.

Lu, Y. and Fang, T., 2014. Examining Personal Air Pollution Exposure, Intake, and Health Danger Zone Using Time Geography and 3D Geovisualization. ISPRS International Journal of Geo-Information, 4 (1), 32-46.

Miller, J.A., 2015. Towards a Better Understanding of Dynamic Interaction Metrics for Wildlife: a Null Model Approach. Transactions in GIS, 19 (3), 342-361.

Nathan, R., Getz, W.M., Revilla, E., Holyoak, M., Kadmon, R., Saltz, D., and Smouse, P.E., 2008. A movement ecology paradigm for unifying organismal movement research. Proceedings of the National Academy of Sciences, 105(49), 19052- 
19059. doi:10.1073/pnas.0800375105

Nathan, R. and Giuggioli, L., 2013. A milestone for movement ecology research. Movement Ecology, 1(1), 1-3. doi:10.1186/2051-3933-1-1

Purves, R.S., Laube, P., Buchin, M., and Speckmann, B., 2014. Moving beyond the point: An agenda for research in movement analysis with real data. Computers, Environment and Urban Systems, 47, 1-4.

Schick, R.S., Loarie, S.R., Colchero, F., Best, B.D., Boustany, A., Conde, D. a., Halpin, P.N., Joppa, L.N., McClellan, C.M., and Clark, J.S., 2008a. Understanding movement data and movement processes: Current and emerging directions. Ecology Letters, 11 (12), 1338-1350.

Soleymani, A., Cachat, J., Robinson, K., Dodge, S., Kalueff, A., and Weibel, R., 2014. Integrating cross-scale analysis in the spatial and temporal domains for classification of behavioral movement. Journal of Spatial Information Science, 8 (8), $1-25$.

Song, Y. and Miller, H.J., 2014. Simulating visit probability distributions within planar space-time prisms. International Journal of Geographical Information Science, 28 (1), 104-125.

Technitis, G., Safi, K., and Weibel, R., 2015. From A to B , randomly: An endpoint-toendpoint random trajectory generator for animal movement. International Journal Geographical Information Science, 29 (6), 912-934.

Theophilides, C.N., Ahearn, S.C., Binkowski, E.S., Paul, W.S., and Gibbs, K., 2006. First evidence of West Nile virus amplification and relationship to human infections. International Journal of Geographical Information Science, 20 (1), $103-115$.

Torrens, P.M., Nara, A., Li, X., Zhu, H., Griffin, W. a., and Brown, S.B., 2012. An extensible simulation environment and movement metrics for testing walking behavior in agent-based models. Computers, Environment and Urban Systems, 36 (1), 1-17.

Wood, O., Visualizing, J., Wood, J., Slingsby, A., and Dykes, J., 2011. City Research Online Visualizing the Dynamics of London's Bicycle Hire Scheme. Cartographica, 46 (4), 239-251.

Xavier, G. and Dodge, S., 2014. An Exploratory Visualization Tool for Mapping the Relationships between Animal Movement and the Environment. In: Proceedings of the 2nd ACM SIGSPATIAL Workshop on MapInteraction. 36-42.

Zhang, Q., Slingsby, a., Dykes, J., Wood, J., Kraak, M.-J., Blok, C. a., and Ahas, R., 2013. Visual analysis design to support research into movement and use of space in Tallinn: A case study. Information Visualization, 13 (3), 213-231. 